Provided for non-commercial research and education use. Not for reproduction, distribution or commercial use.

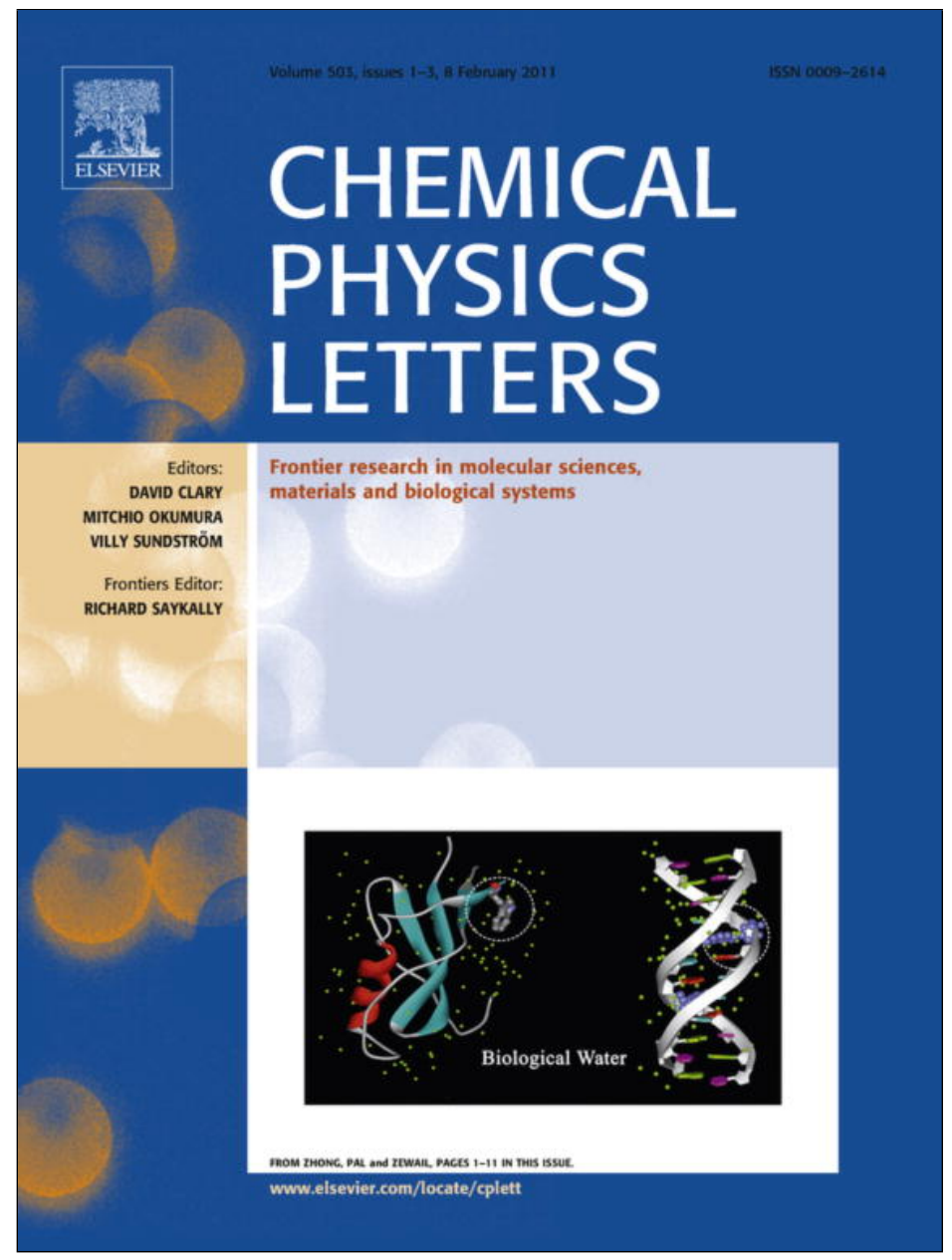

This article appeared in a journal published by Elsevier. The attached copy is furnished to the author for internal non-commercial research and education use, including for instruction at the authors institution and sharing with colleagues.

Other uses, including reproduction and distribution, or selling or licensing copies, or posting to personal, institutional or third party websites are prohibited.

In most cases authors are permitted to post their version of the article (e.g. in Word or Tex form) to their personal website or institutional repository. Authors requiring further information regarding Elsevier's archiving and manuscript policies are encouraged to visit:

http://www.elsevier.com/copyright 


\title{
Perturbation theory and the two-level approximation: A corollary and critique
}

\author{
David L. Andrews*, David S. Bradshaw, Matt M. Coles \\ School of Chemistry, University of East Anglia, Norwich NR4 7TJ, United Kingdom
}

\section{A R T I C L E I N F O}

\section{Article history:}

Received 14 October 2010

In final form 20 December 2010

Available online 22 December 2010

\begin{abstract}
A B S T R A C T
This analysis addresses the use of a two-level approximation to simplify expressions derived from perturbation theory. It is shown that the limitations of validity for the emergent results are more stringent than is commonly understood, being equivalent in effect to the adoption of a more extensive approximation one that significantly undermines the perturbative origin of those expressions. Effectively truncating the completeness relation, a series of interconnected operator relations comes into play, some with physically untenable consequences. A new theorem on the expectation values of operator functions highlights additional constraints upon any molecule modelled as a two-level system.
\end{abstract}

(C) 2010 Elsevier B.V. All rights reserved.

\section{Introduction}

In the development of theory to address the interactions of light and matter, one of the most widely deployed models is the twolevel approximation. Usually applied to electronic states in systems with discrete energy levels, it is a theoretical model that is deeply embedded in a wide range of material representations, extending from those of atoms $[1,2]$ and more recently quantum dots, $[3,4]$ through to a formalism applicable to molecules and chromophores of significant structural complexity [5]. To the extent that any such system is potentially amenable to a two-level representation, the advantages are obvious; relative calculational simplicity, and results cast in formulae that entail a sufficiently small set of parameters for their experimental determination to be realistic. Moreover, the theoretical constructs of a two-level system have a certain appeal, offering the potential to exploit mathematical congruence with the dynamics of a spin- $1 / 2$ system and its associated Pauli matrices, now commonly deployed in schemes for quantum computing.

The quantum mechanics of systems limited to two discrete energy levels is part of a broader field of applications for which the Hilbert space has two degrees of freedom. A complete basis for this space therefore consists of two independent states; a classic example is the inversion of ammonia [6], for which the two basis states are of equal energy, being distinguished by mirror-inverse nuclear geometries. More commonly - and especially within the province of nonlinear optics - two-state calculations are based on selecting two electronic levels, from the many that emerge from the quantum mechanics of any realistic three-dimensional system; these are generally the ground state and one excited state - usually, but not necessarily, the two lowest in energy. In contrast to genuine two-state systems such as ammonia, which in consequence

\footnotetext{
* Corresponding author. Fax: +44 1603592003.

E-mail address: d.l.andrews@uea.ac.uk (D.L. Andrews).
}

display well-characterized Rabi oscillations, most two-level applications are contrived within a perturbation theory approach, where applicability is less plausible.

At the levels of light intensity associated with continuous-wave or relatively weak pulsed laser light, individual atoms or molecules will interact with photons singly. Two-level theory can be very effective in such situations - especially for atoms, leading for example to applications in cavity quantum electrodynamics [7]. It is nonetheless salutary to note that relatively few atomic transitions can legitimately be regarded as two-level systems [8], even in the alkali metals with one electron outside an inert gas configuration core; moreover, Barton long ago pointed out the deficiencies of a two-level model for calculating the frequency shifts of atoms near an interface [9]. In the more recent context of quantum information theory, issues of similarity between two-level atoms and qubit operation have been addressed by Bialynicki-Birula and Sowiński [10] - clearly such models are appropriate only in 'one-electron' ( $\operatorname{spin} \pm 1 / 2$ ) systems.

In the course of developing the received theory of nonlinear optics, the two-level approach has also been widely used. The issues are clearly different from those of the low-intensity dynamics of simple atomic or atom-like systems. With the typical intensity levels of pulsed laser light, there arises a significant probability for two or more photons to interact simultaneously (within the limits of quantum uncertainty) with each optically distinct center. Although the materials that are most effective for the utilization of optical nonlinearity in frequency conversion (especially second harmonic generation, SHG) are those whose energy level structures are significantly more complex than atoms, the two-level approximation has received wide application in such a context $[5,11-18]$; it not only delivers results of a relatively simple form, it also relates well to long-established concepts of chemical structure. A wealth of synthetic studies have built on the anticipated and oft-proven connection between 'push-pull' chromophore structures [19-22] 
(facilitating intramolecular electron transfer) and an enhanced second harmonic response. The devising of simple structural rules, ultimately derived from the two-level model, has assisted the laboratory development of many high-efficiency nonlinear optical materials. On the other hand, the complexities of optical susceptibility calculations based on direct $a b$ initio calculation are such that many studies resort to static perturbation methods, giving results that lack the crucial dispersion characteristics [23-27].

In this Letter, we note additional caveats that should be observed when utilizing optical response calculations limited to two-level implementation. Following a brief summary of the methodology most widely deployed in such calculations, it is shown that there are little-known corollaries - beyond the cautionary principles that have been presented by many others, see for example Refs. [28-30]. In particular, there are significant implications of a theorem that addresses the expectation values of operator analytic functions. Examples are drawn from two areas; broad applications to two-level systems with single-electron wavefunctions, and the nonlinear susceptibility (hyperpolarizability) response of materials engaged in SHG.

\section{Using perturbation theory with the two-level approximation}

The rate of an electromagnetic interaction determined from Fermi's Rule [31] is cast in terms of a quantum amplitude $M_{F I}$ that couples the initial and final states. Time-dependent perturbation theory is generally required to secure an expression for $M_{F I}$ from the following infinite series [32];

$$
\begin{aligned}
M_{F I}= & \sum_{p=0}^{\infty}\left\langle F\left|H_{\text {int }}\left(T_{0} H_{\text {int }}\right)^{p}\right| I\right\rangle=\left\langle F_{\text {int }}+H_{\text {int }} T_{0} H_{\text {int }}+H_{\text {int }} T_{0} H_{\text {int }} T_{0} H_{\text {int }}\right. \\
& +H_{\text {int }} T_{0} H_{\text {int }} T_{0} H_{\text {int }} T_{0} H_{\text {int }}+\cdots|I\rangle
\end{aligned}
$$

Here, $|I\rangle$ and $|F\rangle$ respectively represent the initial and final system states, comprising molecular and radiation parts, and $H_{\text {int }}$ is the interaction operator; in a quantum electrodynamical representation this acts upon both matter and radiation states. Moreover $T_{0} \approx\left(E_{I}-H_{0}\right)^{-1}$, where $H_{0}$ is the unperturbed system Hamiltonian and $E_{I}$ is the initial state energy. The development of Eq. (1) usually involves implementation of the completeness relation $\sum_{R}|R\rangle\langle R|=1$ etc., so that the equation is recast as;

$$
\begin{aligned}
M_{F I}= & \left\langle F\left|H_{\text {int }}\right| I\right\rangle+\sum_{R} \frac{\left\langle F\left|H_{\text {int }}\right| R\right\rangle\left\langle R\left|H_{\text {int }}\right| I\right\rangle}{\left(E_{I}-E_{R}\right)} \\
& +\sum_{R, S} \frac{\left\langle F\left|H_{\text {int }}\right| S\right\rangle\left\langle S\left|H_{\text {int }}\right| R\right\rangle\left\langle R\left|H_{\text {int }}\right| I\right\rangle}{\left(E_{I}-E_{R}\right)\left(E_{I}-E_{S}\right)} \\
& +\sum_{R, S, T} \frac{\left\langle F\left|H_{\text {int }}\right| T\right\rangle\left\langle T\left|H_{\text {int }}\right| S\right\rangle\left\langle S\left|H_{\text {int }}\right| R\right\rangle\left\langle R\left|H_{\text {int }}\right| I\right\rangle}{\left(E_{I}-E_{R}\right)\left(E_{I}-E_{S}\right)\left(E_{I}-E_{T}\right)}+\ldots,
\end{aligned}
$$

where the virtual system states are denoted by $|R\rangle,|S\rangle,|T\rangle \ldots$ upon which $H_{0}$ operates - and $E_{n}$ is the energy of the state denoted by its subscript.

Following a development of Eq. (2) in a complete basis set of radiation and atomic/molecular states, results for the description of optical response in a two-level approximation are commonly secured from the appropriate contribution to the series expansion; a process involving $n$ photons is generally described by the $n$th term. However, the same results emerge if the Hilbert sub-space for the molecular states is limited, at this initial stage, to the two chosen energy levels, a ground state $|0\rangle$ and a single excited state $|1\rangle$. Such a strategy is exactly equivalent to effecting the completeness relation in the form $|0\rangle\langle 0|+| 1\rangle\langle 1|=1$, clearly an approximation as other state projections are excluded from the summation. Formally the two-level version of the completeness relation can be justified in the following manner:

$$
\begin{aligned}
1 & \equiv \sum_{R}|R\rangle\left\langle R\left|\equiv \sum_{\rho, r}\right| \rho_{\text {rad }}\right\rangle\left|r_{\text {mol }}\right\rangle\left\langle r_{\text {mol }}\right|\left\langle\rho_{\text {rad }}\right| \equiv \mathbf{1}_{\text {rad }} \times \mathbf{1}_{\text {mol }} \\
& \equiv \mathbf{1}_{\text {rad }} \times\left\{|0\rangle\langle 0|+| 1\rangle\left\langle 1\left|+\sum_{r \notin\{0,1\}}\right| r\right\rangle\langle r|\right\}
\end{aligned}
$$

For example, application of the two-level approximation to SHG representing one widely employed adoption of the model in nonlinear optics, gives the following result for the hyperpolarizability tensor, $\beta_{i j k}^{00}$, drawn from the third term on the right-hand side of Eq. (2);

$$
\begin{aligned}
\beta_{i j k}^{00}(-2 \omega ; \omega, \omega)= & \sum_{r_{1}, r_{2} \in 0,1}\left(\frac{\mu_{i}^{0 r_{2}} \mu_{j}^{r_{2} r_{1}} \mu_{k}^{r_{1} 0}}{\left(\tilde{E}_{r_{2} 0}-2 \hbar \omega\right)\left(\tilde{E}_{r_{1} 0}-\hbar \omega\right)}\right. \\
& \left.+\frac{\mu_{j}^{0 r_{2}} \mu_{i}^{r_{2} r_{1}} \mu_{k}^{r_{1} 0}}{\left(\tilde{E}_{r_{2} 0}+\hbar \omega\right)\left(\tilde{E}_{r_{1} 0}-\hbar \omega\right)}+\frac{\mu_{j}^{0 r_{2}} \mu_{k}^{r_{2} r_{1}} \mu_{i}^{r_{1} 0}}{\left(\tilde{E}_{r_{2} 0}+h \omega\right)\left(\tilde{E}_{r_{1} 0}+2 \hbar \omega\right)}\right) .
\end{aligned}
$$

Here, the index $i$ is assigned to emission and $j, k$ to absorption; $r_{1}$ and $r_{2}$ represent intermediate matter states, $\tilde{E}_{r 0} \equiv \tilde{E}_{r}-\tilde{E}_{0}$ is an energy difference, $\mu^{0 r} \equiv\langle 0|\mu| r\rangle$ denotes a transition dipole moment, and $h \omega$ is the energy of an input photon. Furthermore, the tildes denote the inclusion of a damping term $i \gamma$, where $2 \gamma$ is the FWHM line-width. Physical insights into the severity of such an approximation, limiting the intermediate states to either the ground or the single excited state, can be drawn by consideration of implications that emerge from the development of theory in the next section.

\section{The expectation values of operators in the two-level model}

We now explore in more detail the consequences of using standard quantum mechanical operator constructs within the context of an artificially truncated basis set. In particular, new insights can be drawn from considering the implications of the two levels, though differing in energy, nonetheless delivering identical expectation values with respect to another quantum operator. Examples are rife; an illustration is readily drawn from the electron dynamics of atomic and molecular systems, in which the expectation value of linear vector momentum is commonly zero for all eigenstates of the Schrödinger operator. We shall show that, within the two-level approximation, an equality between the expectation values of a particular operator in the two states has a much broader consequence, viz.;

$\langle 0|\hat{A}| 0\rangle=\langle 1|\hat{A}| 1\rangle$

$\Rightarrow\langle 0|f(\hat{A})| 0\rangle=\langle 1|f(\hat{A})| 1\rangle$

where $f(\hat{A})$ is any analytic function, and $\hat{A}$ is any Hermitian operator. Consideration is then given to a brief exploration of the physical implications, with particular regard to the energies and forms of charge distribution of the two states.

\subsection{Theorem}

Any analytic function can be written in the form:

$$
f(\hat{A})=\sum_{n=0}^{\infty} \frac{f^{(n)}(c)}{n !}(\hat{A}-c)^{n}
$$

where $c$ is a constant. Thus:

$$
\begin{aligned}
\langle 1|f(\hat{A})| 1\rangle-\langle 0|f(\hat{A})| 0\rangle & =\left\langle 1\left|\sum_{n=0}^{\infty} \frac{f^{(n)}(c)}{n !}(\hat{A}-c)^{n}\right| 1\right\rangle \\
& -\left\langle 0\left|\sum_{n=0}^{\infty} \frac{f^{(n)}(c)}{n !}(\hat{A}-c)^{n}\right| 0\right\rangle \\
& =\sum_{n=0}^{\infty} \frac{f^{(n)}(c)}{n !}\left[\left\langle 1\left|(\hat{A}-c)^{n}\right| 1\right\rangle-\left\langle 0\left|(\hat{A}-c)^{n}\right| 0\right\rangle\right] .
\end{aligned}
$$


To establish the sought relationship, it suffices to show that the term in square brackets on the right-hand side of (8) vanishes. First, we write:

$$
\begin{aligned}
\left\langle 1\left|(\hat{A}-c)^{n}\right| 1\right\rangle-\left\langle 0\left|(\hat{A}-c)^{n}\right| 0\right\rangle= & \left\langle 1\left|(\hat{A}-c) \cdot 1 \cdot(\hat{A}-c)^{n-1}\right| 1\right\rangle \\
& -\left\langle 0\left|(\hat{A}-c)^{n-1} \cdot 1 \cdot(\hat{A}-c)\right| 0\right\rangle .
\end{aligned}
$$

Using the completeness relation for the two-level approximation, i.e. $|0\rangle\langle 0|+| 1\rangle\langle 1|=1$, we find from the right-hand side of (9) that:

$$
\begin{aligned}
& \underbrace{\langle 1|(\hat{A}-c)| 0\rangle\left\langle 0\left|(\hat{A}-c)^{n-1}\right| 1\right\rangle-\left\langle 0\left|(\hat{A}-c)^{n-1}\right| 1\right\rangle\langle 1|(\hat{A}-c)| 0\rangle}_{\quad}=0 \\
& \quad+\langle 1|(\hat{A}-c)| 1\rangle\left\langle 1\left|(\hat{A}-c)^{n-1}\right| 1\right\rangle-\left\langle 0\left|(\hat{A}-c)^{n-1}\right| 0\right\rangle\langle 0|(\hat{A}-c)| 0\rangle \\
& \quad \equiv(\hat{A}-c)_{11}(\hat{A}-c)_{11}^{n-1}-(\hat{A}-c)_{00}^{n-1}(\hat{A}-c)_{00}
\end{aligned}
$$

Hence;

$$
\begin{aligned}
(\hat{A}-c)_{11}^{n}-(\hat{A}-c)_{00}^{n} & =(\hat{A}-c)_{11}(\hat{A}-c)_{11}^{n-1}-(\hat{A}-c)_{00}^{n-1}(\hat{A}-c)_{00} \\
& =(\hat{A}-c)_{00}\left[(\hat{A}-c)_{11}^{n-1}-(\hat{A}-c)_{00}^{n-1}\right],
\end{aligned}
$$

since $(\hat{A}-c)_{00}=(\hat{A}-c)_{11}$. The case $n=2$ delivers a vanishing result, by virtue of the initial assumption; the result for arbitrary $n$ follows by induction. Thus we have verified that, if $\langle 0|\hat{A}| 0\rangle=\langle 1|\hat{A}| 1\rangle$, then the two-level approximation requires that $\left\langle 0\left|\hat{A}^{n}\right| 0\right\rangle=\left\langle 1\left|\hat{A}^{n}\right| 1\right\rangle$. By an analogous method, it emerges that if a vector operator $\hat{\mathbf{A}}$ (such as the electric dipole moment operator $\hat{\mu}$ ) has identical expectation values in the two basis states, then so too will the expectation values of $\hat{A}_{i} \hat{A}_{j}$, and hence by extension the expectation values of any string of components $\hat{A}_{i} \hat{A}_{j} \ldots \hat{A}_{n}$.

\subsection{Physical implications}

A clear flaw in the two-level approximation is most readily and simply exhibited by a system in which the ground and singleelectron excited levels have the same expectation values for the linear vector momentum of the electron, $\langle\mathbf{p}\rangle_{\mathbf{1}}=\langle\mathbf{p}\rangle_{\mathbf{0}}$, (those values usually being zero, in both instances). This leads to a plainly absurd conclusion that $\left\langle\mathbf{p}^{2}\right\rangle_{1}=\left\langle\mathbf{p}^{2}\right\rangle_{0}$, i.e. the expectation value for the squares of momentum - and hence that the kinetic energies $T$ should also be the same for both energy levels. Moreover, if the associated potential energy, $V$, takes the centric form of an $m$ th power dependence on displacement, then the Virial Theorem dictates that $2\langle T\rangle=m\langle V\rangle$, so that with $E=\langle T\rangle+\langle V\rangle$ it would have to be inferred that $E_{1}=E_{0}$, implying that the two states are a degenerate pair. The patently nonsensical result arises as a result of combining an application of the completeness relation, limited in compass to two specified states, alongside the commonly satisfied condition that those two states should have the same expectation value for one particular observable - in this case electron momentum. It is readily verified that, pursuing the analysis for such a pair of states, but using the completeness relation without restriction, removes the implication of a common energy and resolves the conundrum.

\section{Two-level approximation in nonlinear optics}

We now resume consideration of the two-level calculations relating to second harmonic generation, representative of an application in nonlinear optics. From the earlier general expression, a result identified with the two-level approximation is readily determined by an algorithmic method [32-34]. This again involves the restriction of both intermediate states featured within Eq. (4) to $|0\rangle$ and $|1\rangle$. Only four unique routes can describe transitions starting and finishing in the ground molecular state progressing through both $r_{1}$ and $r_{2}$; explicitly the $0 \rightarrow r_{1} \rightarrow r_{2} \rightarrow 0$ sequences are expressible as the binary sequences $0000,0100,0010$ and 0110. Each generates a combination of both transition dipole mo- ments, either $\mu^{01}$ or $\mu^{10}$, as well as the static dipole moments of the ground and excited energy levels, $\mu^{00}$ and $\mu^{11}$, respectively. Detailed analysis of nonlinear optical susceptibilities shows that a dependence on static moments always emerges in terms of their vector difference with respect to the ground state, here $d=\mu^{11}-\mu^{00}$, with the following prescription an expedient to deliver correct results: $\mu^{11} \rightarrow \mu^{11}-\mu^{00}=d ; \mu^{00} \rightarrow 0$. The validity of this algorithm can be proven by performing a canonical transformation on the multipolar coupling Hamiltonian [35]. Application of this prescription dictates that any sequence connecting the initial and final states through a ground state static dipole is discarded; hence in the case of SHG only one of the four sequences, namely 0110 , persists. It is then clear that the hyperpolarizability tensor becomes;

$$
\begin{aligned}
\beta_{i j k}^{00}= & \frac{\mu_{i}^{01} d_{j} \mu_{k}^{01}}{\left(\tilde{E}_{10}-2 h \omega\right)\left(\tilde{E}_{10}-h \omega\right)}+\frac{\mu_{j}^{01} d_{i} \mu_{k}^{01}}{\left(\tilde{E}_{10}+h \omega\right)\left(\tilde{E}_{10}-h \omega\right)} \\
& +\frac{\mu_{j}^{01} d_{k} \mu_{i}^{01}}{\left(\tilde{E}_{10}+h \omega\right)\left(\tilde{E}_{10}+2 h \omega\right)} .
\end{aligned}
$$

The result represented by Eq. (12) is frequently used in support of a strategy for devising nonlinear optical materials, on the premise that a non-zero value for $\boldsymbol{d}$ is necessary to support a finite hyperpolarizability.

In general, for an $n$-photon parametric process, each state sequence generated in deploying the algorithm can be written in binary notation as $0 r_{1} r_{2} \ldots r_{n-1} 0$, where $r \in\{0,1\}$. For odd $n$, sequences commencing and ending in a zero necessarily accommodate at least one element ' 11 ' (without such an element, the same binary string would have to include ' 00 ', and therefore represent a term to be discarded on the basis of the above prescription). Since inclusion of the element ' 11 ' generates a dependence on the dipole difference $\boldsymbol{d}$, it then follows that each term in the nonlinear susceptibility - and hence the full result - must vanish if $\boldsymbol{d}$ is zero, in the two-level approximation. However, for even- $n$ processes (e.g. third or fifth harmonic generation), it is clear that the alternating binary string $0101 \ldots 0$ will always arise and generate a contribution to the nonlinear response, even in materials with vanishing d. Furthermore, from the theorem established in Section 3, it now emerges that when $\boldsymbol{d}$ is zero, then $\left\langle\mu^{2}\right\rangle_{11}-\left\langle\mu^{2}\right\rangle_{00}$ should also vanish. If we take the variance of $\mu$ to be $\Delta \bar{\mu}^{2}=\left\langle\mu^{2}\right\rangle-\langle\mu\rangle^{2}$, it then becomes apparent that the two-level approximation requires fluctuations in $\mu$ to be identical in the two energy levels. Thus, there are further, hidden constraints imposed upon the possible axial forms of electronic distribution that a two-level molecule may legitimately take.

\section{Discussion}

It is widely recognized that any use of a two-level approximation is primarily a matter of simplification at the expense of precision and breadth of applicability. For example, in particular connection with the illustrative case considered in the preceding analysis, the nonlinear susceptibility that supports second harmonic generation can certainly be supported in high-symmetry materials with neither ground nor excited state dipole, provided the point or space group supports an electric octupole - systems of $D_{3 h}$ or $T_{d}$ symmetry for example [36]. There is extensive literature on the development of such chromophores [37-40], particularly in the many pioneering studies of Zyss et al. With these systems it is understood that the existence of octupolar nonlinearities points to inadequacies of the traditional two-level model, often demanding the use of models based on a more accurate threelevel representation [41]. 
It is appropriate to note certain other caveats and corollaries, regarding the two-level model, that have been previously pointed out. An example is afforded by recent work that has identified a rule dictating that the sum over all states of optical susceptibilities of any order has to be zero [42]. The result emerges in the form of a traceless operator in Hilbert space, symbolized by $\sum_{r}\langle r|\hat{A}| r\rangle=0$, where $r$ denotes a matter state and the operator $\hat{A}$ is here to be identified with the operator form of the polarizability $\hat{\alpha}_{i j}$, hyperpolarizability $\hat{\beta}_{i j k}$ or higher-order response tensor. The result is exact and widely applicable; however with the two-level model it imposes a rarely observed corollary - that components of the excited state response tensors are precisely the negative of the corresponding ground state components [34].

Applicability issues have also been considered in connection with studies of the relationship between the minimal-coupling

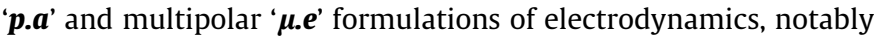
addressed and resolved in a series of works by Power and Thirunamachandran $[43,44]$ and Woolley $[45,46]$. In the context of multiphoton absorption, Meath and Power demonstrated that the twolevel approximation is indeed never valid when deployed with a minimal-coupling Hamiltonian, since this kind of formulation requires sum rules to be applied over all levels in order to secure a usable result for the quantum amplitude [12]. It is interesting to reflect that the results of the theorem delivered in the present paper apply to both forms of coupling, addressing the matrix elements of both momentum and electric dipole operators.

To conclude, the pervasive usage of two-level approximations appears to suggest a need for the full implications of such models to be better understood, particularly when applied within the framework of perturbation theory; this has been the purpose of the present analysis. Two-level models should be utilized in full cognizance of the limitations on their validity, associated with the consequences of effectively truncating the completeness relation. By proving a new theorem on the expectation values of operator functions, we have shown that a series of interconnected operator relations comes into play - and that the unguarded application of two-level results can have physically untenable consequences.

\section{Acknowledgments}

The authors would like to thank Professors Mohamed Babiker and William J. Meath for helpful discussions, and EPSRC for funding this research.

\section{References}

[1] L. Allen, J.H. Eberly, Optical Coherence and Two-Level Atoms, Dover, Mineola NY, 1987.

[2] B.W. Shore, Theory of Coherent Atomic Excitation (vol. 1), Wiley-Interscience, New York, 1995. Chapter 3.
[3] A. Zrenner, E. Beham, S. Stufler, F. Findeis, M. Bichler, G. Abstreiter, Nature 418 (2002) 612.

[4] M.S. Skolnick, D.J. Mowbray, Annu. Rev. Mater. Res. 34 (2004) 181

[5] S. Mukamel, Principles of Nonlinear Optical Spectroscopy, Oxford University Press, New York, 1995. pp. 189.

[6] R. Feynman, Feynman Lectures on Physics (vol. 3), Addison-Wesley Longman, Reading, MA, 1970. Sec. 9-1.

[7] M. Brune, S. Haroche, in: G.-L. Oppo, S.M. Barnett, E. Riis, M. Wilkinson (Eds.) Quantum Dynamics of Simple Systems, Institute of Physics, Bristol, 1994, pp. 49-70.

[8] S.C. Rand, Lectures on Light. Nonlinear and Quantum Optics using the Density Matrix, University Press, Oxford, 2010. p. 67.

[9] G. Barton, J. Phys. B: At. Mol. Phys. 7 (1974) 2134

[10] I. Bialynicki-Birula, T. Sowiński, Phys. Rev. A 76 (2007) 062106.

[11] J.L. Oudar, D.S. Chemla, J. Chem. Phys. 66 (1977) 2664.

[12] B. Dick, G. Hohlneicher, J. Chem. Phys. 76 (1982) 5755.

[13] W.J. Meath, E.A. Power, J. Phys. B: At. Mol. Opt. Phys. 17 (1984) 763.

[14] R. Wortmann, P. Krämer, C. Glania, S. Lebus, N. Detzer, Chem. Phys. 173 (1993) 99.

15] D.L. Andrews, W.J. Meath, J. Phys. B: At. Mol. Opt. Phys. 26 (1993) 4633.

[16] B.N. Jagatap, W.J. Meath, Chem. Phys. Lett. 258 (1996) 293.

[17] M. Drobizhev, F. Meng, A. Rebane, Y. Stepanenko, E. Nickel, C.W. Spangler, J Phys. Chem. B 110 (2006) 9802.

[18] R.W. Boyd, Nonlinear Optics, Academic Press, Amsterdam, 2008. p. 277.

[19] F. Terenziani, S. Ghosh, A.-C. Robin, P.K. Das, M. Blanchard-Desce, J. Phys. Chem. B 112 (2008) 11498.

[20] A. Corozzi, B. Mennucci, R. Cammi, J. Tomasi, J. Phys. Chem. A 113 (2009) 14774

[21] F. Quist, C.M.L. Vande Velde, D. Didier, A. Teshome, I. Asselberghs, K. Clays, S. Sergeyev, Dyes Pigments 81 (2009) 203.

[22] J. Rotzler, D. Vonlanthen, A. Barsella, A. Boeglin, A. Fort, M. Mayor, Eur. J. Org. Chem. (2010) 1096

[23] S. Sharma, P. Gangopadhyay, A. Swathi, T.P. Radhakrishnan, Phys. Chem. Chem. Phys. 2 (2000) 1147.

[24] Y. Zhang, W. Zhu, W. Wang, H. Tian, J. Su, W. Wang, J. Mater. Chem. 12 (2002) 1294.

[25] H. Hou, Y. Wei, Y. Song, Y. Zhu, L. Li, Y. Fan, J. Mater. Chem. 12 (2002) 838.

[26] K. Ohta, S. Yamada, T. Tanaka, K. Kamada, Mol. Phys. 101 (2003) 315.

27] M. Nakano et al., Chem. Phys. Lett. 467 (2008) 120.

[28] D.R. Reichman, R.J. Silbey, J. Chem. Phys. 104 (1996) 1506.

[29] M. Frasca, Annu. Phys. 306 (2003) 193.

[30] J. Cheng, J. Zhou, Phys. Rev. A 67 (2003) 041404.

[31] L. Mandel, E. Wolf, Optical Coherence and Quantum Optics, University Press, Cambridge, U.K., 1995. p. 871.

[32] L.C. Dávila Romero, D.L. Andrews, J. Phys. B: At. Mol. Opt. Phys. 32 (1999) 2277

[33] D.L. Andrews, L.C. Dávila Romero, W.J. Meath, J. Phys. B: At. Mol. Opt. Phys. 32 (1999) 1.

[34] D.L. Andrews, L.C. Dávila Romero, J. Phys. B: At. Mol. Opt. Phys. 34 (2001) 2177.

[35] G. Juzeliūnas, L.C. Dávila Romero, D.L. Andrews, Phys. Rev. A 68 (2003) 043811.

[36] D.L. Andrews, P. Allcock, Optical Harmonics in Molecular Systems, Wiley-VCH, Weinheim, Germany, 2002. Appendix 4.

[37] M. Moreno Oliva, J. Casado, J.T. López Navarrete, G. Hennrich, S. van Cleuvenbergen, I. Asselberghs, K. Clays, M.C. Ruiz Delgado, J.-L. Brédas, J.S. Seixas de Melo, L. De Cola, Chem. Eur. J. 15 (2009) 8223.

[38] M. Quintiliani, J. Pérez-Moreno, I. Asselberghs, P. Vázquez, K. Clays, T. Torres, J. Phys. Chem. B 114 (2010) 6309.

[39] A. Valore et al., J. Am. Chem. Soc. 132 (2010) 4966

[40] B.J. Coe et al., J. Am. Chem. Soc. 132 (2010) 3496.

[41] J. Zyss, J. Chem. Phys. 98 (1993) 6583.

[42] L.C. Dávila Romero, D.L. Andrews, J. Opt. B: Quantum Semiclass. Opt. 6 (2004) 59.

[43] E.A. Power, T. Thirunamachandran, Am. J. Phys. 46 (1978) 370.

[44] E.A. Power, T. Thirunamachandran, Phys. Rev. A 28 (1983) 2649.

[45] R.G. Woolley, Int. J. Quantum Chem. 74 (1999) 531.

[46] R.G. Woolley, Proc. R. Soc. Lond. A 456 (2000) 1803. 